\title{
Molecular Histopathology
}

\author{
Hussein A. Kaoud
}

Additional information is available at the end of the chapter

http://dx.doi.org/10.5772/52919

\section{Introduction}

Traditional pathology concentrates on the morphological manifestations of disease. Molecular pathology, in addition, integrates tools of molecular biology to: isolate and identify the causative agents in infectious disease, understand the role of differential gene expression in disease etiology, provide more accurate means of disease diagnosis and provide more individualized approaches to therapy.

Molecular pathology is a branch of the biomedical sciences which focuses on the progress, development, and evolution of diseases on the molecular level. It can be applied practically to patients in addition to being utilized in biomedical research to learn more about specific diseases, ranging from cancer to genetic conditions. Usually, molecular pathology is treated as a subset of the field of pathology, but it also involves genetics, immunology, and many other aspects of the medical field, and people can approach it from a number of perspectives.

A molecular pathologist can conduct a variety of tests to learn about the fundamental components of a cell, including the array of amino acids which makes up cellular DNA. In addition to performing amino acid sequencing, people in this field also look at samples of cellular tissue, and they perform a variety of tests to learn more about the progress of disease in specific patients and in general.

To understand the causes and molecular basis of the development of disease, with particular reference to cancer, and to apply this knowledge to improving disease prevention, detection, diagnosis and treatment. Improving the outlook for cancer patients can only come from an understanding of molecular and cell biology. There has been a dramatic increase in knowledge of the molecular genetics of cancers over the last few years and already we have reached the point where this can be translated into clinical application.

Many diseases are caused by inherited gene mutations, for example, cystic fibrosis, muscular dystrophy and lysosomal storage diseases. Other gene alterations confer a greater susceptibility to disease - for example, cancer or heart disease. 
One common use for a molecular pathologist is in the study of specimens taken from cancer patients. The pathologist can test the specimen to determine where the cancer originated, and to look for biomarkers which could indicate susceptibility to specific cancer treatments. For example, a breast cancer which is estrogen sensitive will be treated differently than a breast cancer which is not. Using molecular pathology, an oncologist can develop a treatment approach which is tailored to the patient.

Key techniques used in molecular pathology to identify relationships between gene alterations and disease include cell isolation and cell culture. Immunohistochemistry is not considered a molecular technique but it is based on the antigen-antibody affinity, it has emerged as a powerful investigative tool that can provide supplemental information to the routine morphological assessment of tissues. The antibody is usually linked to other molecules to aid visualization [(fluorophore, reporter enzyme, etc), FISH (A cytogenetic method of detecting and localizing specific DNA sequences on chromosomes)] and laboratory molecular biology techniques (identification of gene mutations, expression profiling, protein analysis, blotting, microarrays).

It is thought that the development of malignant disease progresses through defined stages such as hyperplasia, dysplasia, carcinoma in situ, primary carcinoma, invasive carcinoma and metastases, each of which may be linked to mutations and alterations in the expression of subsets of genes. Laser microdissection is useful for isolating particular cells, or populations of cells, from tissue (frozen sections, fixed sections and in cell culture monolayers) for genetic analysis allowing direct comparison of nucleic acid from cells in different stages of disease progression.

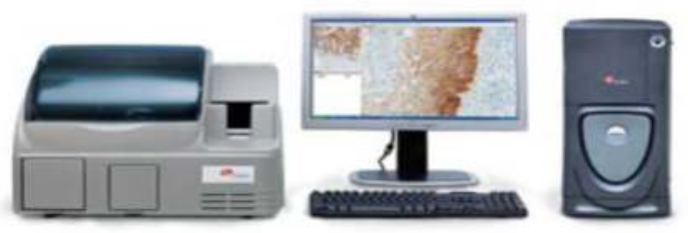

Figure 1. Automated Cellular Imaging System (ACISR III), an example of an image acquisition and image analysis instrument.

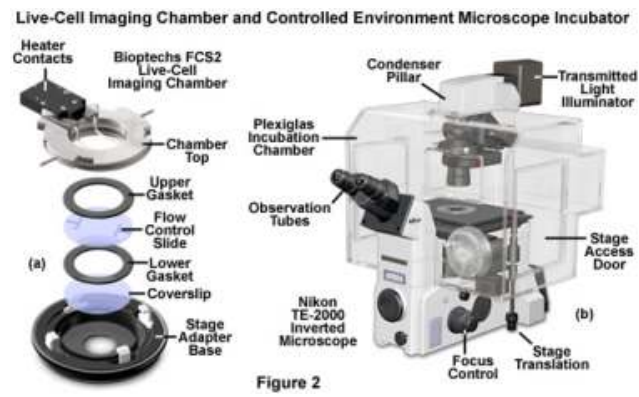

Figure 2. 
The impact of gene alterations on protein function and disease can be investigated using a variety of imaging techniques (Figs.1 and 2). Of particular importance is the imaging of protein-protein interactions using time-lapse imaging, TIRF microscopy and confocal fluorescence microscopy techniques such as 3-D rendering, FRET/BRET. These enable spatial and temporal analysis of dynamic events involving, for example, receptor interactions and other signaling events (Fig.3).

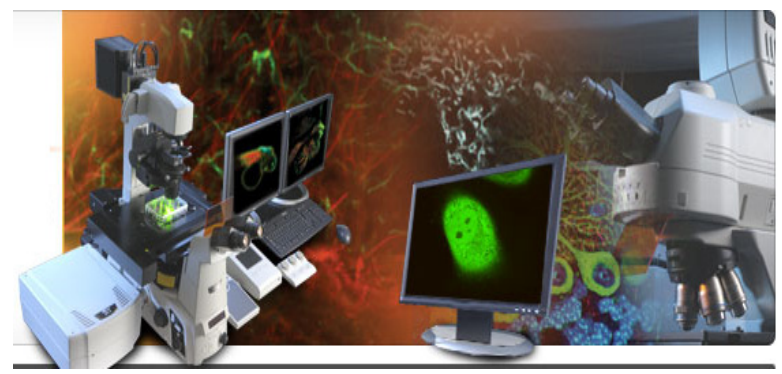

Figure 3. Nikon's powerful fully-automated confocal imaging system, capable of capturing high-quality confocal images of cells and molecular events.

\subsection{Immune system (tumor immunology)}

Another important role of the immune system (Fig.4) is to identify and eliminate tumors. The transformed cells of tumors express antigens that are not found on normal cells. To the immune system, these antigens appear foreign, and their presence causes immune cells to attack the transformed tumor cells. The antigens expressed by tumors have several sources [1] some are derived from oncogenic viruses like human papillomavirus, which causes cervical cancer,[2] while others are the organism's own proteins that occur at low levels in normal cells but reach high levels in tumor cells. One example is an enzyme called tyrosinase that, when expressed at high levels, transforms certain skin cells (e.g. melanocytes) into tumors called melanomas [3,4]. A third possible source of tumor antigens are proteins normally important for regulating cell growth and survival, that commonly mutate into cancer inducing molecules called oncogenes $[1,5,6]$.

The main response of the immune system to tumors is to destroy the abnormal cells using killer $\mathrm{T}$ cells, sometimes with the assistance of helper $\mathrm{T}$ cells [4, 7]. Tumor antigens are presented on MHC class I molecules in a similar way to viral antigens. This allows killer $\mathrm{T}$ cells to recognize the tumor cell as abnormal [8] NK cells also kill tumorous cells in a similar way, especially if the tumor cells have fewer MHC class I molecules on their surface than normal; this is a common phenomenon with tumors [9] .Sometimes antibodies are generated against tumor cells allowing for their destruction by the complement system [5].

Clearly, some tumors evade the immune system and go on to become cancers [10]. Tumor cells often have a reduced number of MHC class I molecules on their surface, thus avoiding detection by killer T cells [8]. Some tumor cells also release products that inhibit the immune response; for example by secreting the cytokine TGF- $\beta$, which suppresses the activity of 
macrophages and lymphocytes [11]. In addition, immunological tolerance may develop against tumor antigens, so the immune system no longer attacks the tumor cells [10].

Paradoxically, macrophages can promote tumor growth [12] when tumor cells send out cytokines that attract macrophages, which then generate cytokines and growth factors that nurture tumor development. In addition, a combination of hypoxia in the tumor and a cytokine produced by macrophages induces tumor cells to decrease production of a protein that blocks metastasis and thereby assists spread of cancer cells.
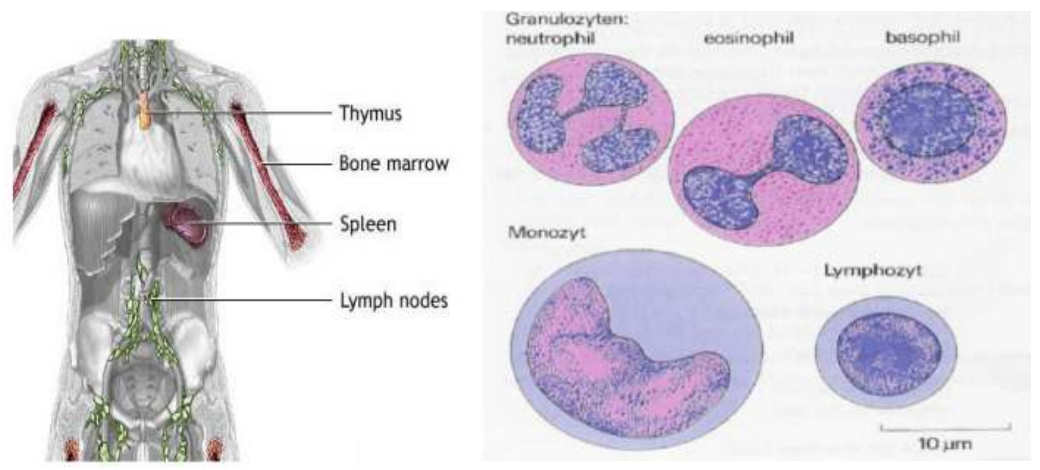

Figure 4. The immune system protects the body from potentially harmful substances. The inflammatory response (inflammation) is part of innate immunity. It occurs when tissues are injured by bacteria, trauma, toxins, heat or any other cause.

\subsection{Genetic diseases (genetic disorders)}

A genetic disorder is an illness caused by abnormalities in genes or chromosomes, especially a condition that is present from before birth. Most genetic disorders are quite rare and affect one person in every several thousands or millions.

A genetic disorder may or may not be a heritable disorder. Some genetic disorders are passed down from the parents' genes, but others are always or almost always caused by new mutations or changes to the DNA. In other cases, the same disease, such as some forms of cancer, may be caused by an inherited genetic condition in some people, by new mutations in other people, and by non-genetic causes in still other people.

\section{Methods in molecular pathology}

Nucleic acid-based testing is becoming a crucial diagnostic tool not only in the setting of inherited genetic disease (e.g., cystic fibrosis and hemochromatosis) but also in a wide variety of hemato-oncologic, solid neoplastic and infectious conditions. Molecular diagnostics provides the necessary underpinnings for any successful application of gene therapy or biologic response modifiers. It offers a great tool for assessing therapy response and detecting minimal residual disease. Currently, it is estimated that more than $5 \%$ of all laboratory testing is based on DNA or RNA analysis. 


\subsection{Tissue microdissection methods}

Microdissection can be performed in a variety of ways, all of which have different advantages and disadvantages. These methods range from the simple and inexpensive manual methods to laser-capture microdissection (LCM) methods that require expensive and complex equipment. These methods are reviewed here, with particular emphasis on manual microdissection, which can be performed easily with common equipment in the pathology laboratory (Fig. 5).

One of the major benefits of microdissection is the production of relatively pure samples of morphologically confirmed cellular populations [13]. This relative purity may be essential for assessing a genetic change or for quantification of genetic changes. For example, in assessing possible loss of heterozygosity of tumor suppressor genes, normal contaminating cells will artifactually equalize the allelic balance, and it may appear that the tumor cells contain normal DNA. In molecular identity testing for tissue floaters (tissue carryover artifacts), microdissection is essential for separating the fragments of the suspected floater from the rest of the tissue sample [14,15]. When examining expression of some gene targets in tumor cells at the mRNA level, results may be confounded by the expression found in normal contaminating stromal and lymphoid cells. In some diseases it may be important to measure events in rare single neoplastic cells [16-18] . Perhaps the most notable example is in Hodgkin lymphoma, where molecular experiments require detailed microdissection to isolate the Reed-Sternberg cells from the surrounding lymphoid infiltrates.

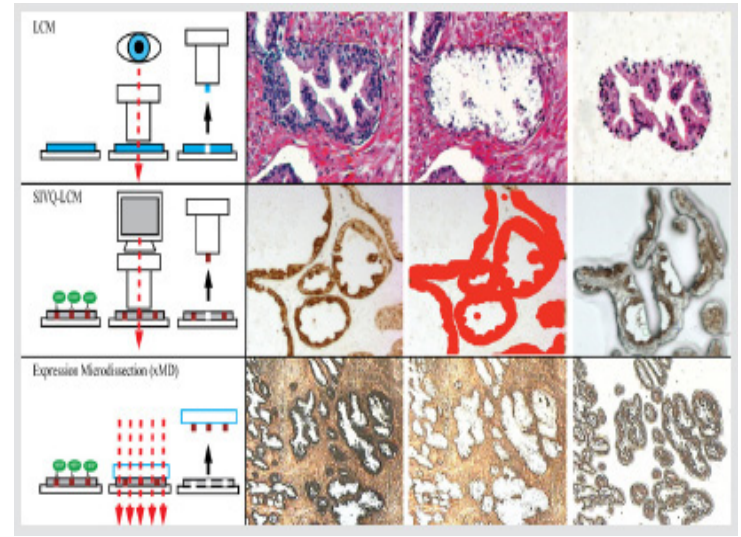

Figure 5. This method "microdissection", can be performed easily with common equipment in the pathology laboratory.

\subsection{Amplification methods}

The polymerase chain reaction (PCR) is a laboratory technique for "amplifying" a specific DNA sequence (Fig.6). PCR is extremely efficient and sensitive; it can make millions or billions of copies of any specific sequence of DNA, even when the sequence is in a complex mixture. Because of this power, researchers can use it to amplify sequences even if they only 
have a minute amount of DNA. A single hair root, or a microscopic blood stain left at a crime scene, for example, contains ample DNA for PCR.

PCR is based on the way cells replicate their DNA. During DNA replication, the two strands of each DNA molecule separate, and DNA polymerase, an enzyme, assembles nucleotides to form two new partner strands for each of the original strands. The original strands serve as templates for the new strands. The new strands are assembled such that each nucleotide in the new strand is determined by the corresponding nucleotide in the template strand. The nucleotides adenine (A) and thymine (T) always lie opposite each other, as do cytosine (C) and guanine $(\mathrm{G})$. Because of this base-pairing specificity, each newly synthesized partner strand has the same sequence as the original partner strand, and replication produces two identical copies of the original double-stranded DNA molecule.

In PCR, a DNA sequence that a researcher wants to amplify, called the "target" sequence, undergoes about thirty rounds of replication in a small reaction tube. During each replication cycle, the number of molecules of the target sequence doubles, because the products and templates of one round of replication all become the templates for the next round. After $\mathrm{n}$ rounds of replication, $2^{\mathrm{n}}$ copies of the target sequence are theoretically produced. After thirty cycles, PCR can produce $2^{30}$ or more than ten billion copies of a single target DNA sequence. This is called a polymerase chain reaction because DNA polymerase catalyzes a chain reaction of replication.
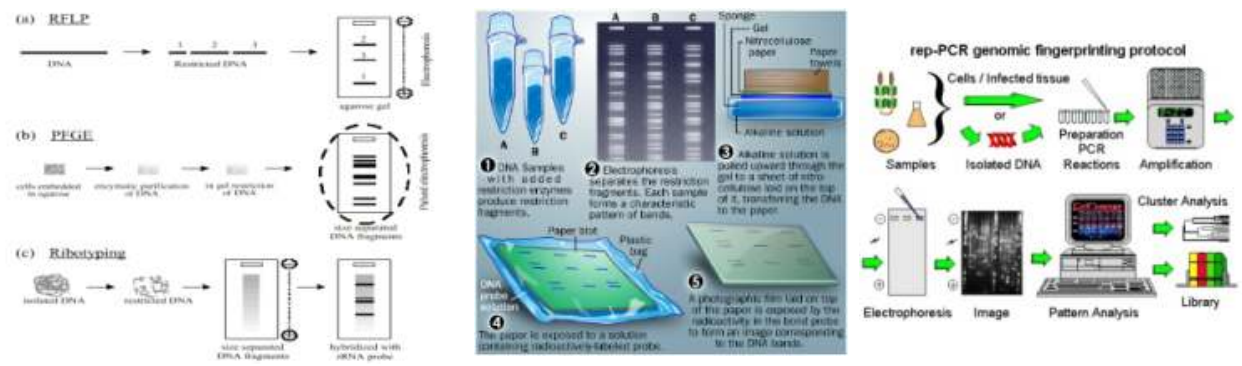

Figure 6. PCR is used to enlarge a few pieces of DNA which would create thousands to millions of copies of that one sample of DNA.

\subsection{Gel electrophoresis methods}

Gel electrophoresis is a method used in clinical chemistry to separate proteins by charge and or size (IEF agarose, essentially size independent) and in biochemistry and molecular biology to separate a mixed population of DNA and RNA fragments by length, to estimate the size of DNA and RNA fragments or to separate proteins by charge [19]. Nucleic acid molecules are separated by applying an electric field [20] to move the negatively charged molecules through an agarose matrix. Shorter molecules move faster and migrate farther than longer ones because shorter molecules migrate more easily through the pores of the gel. This phenomenon is called sieving. Proteins are separated by charge in agarose because the pores of the gel are too large to sieve proteins. Gel electrophoresis can also be used for separation of nanoparticles. 
Gel electrophoresis uses a gel as an anticonvective medium and or sieving medium during electrophoresis, the movement of a charged particle in an electrical field. Gels suppress the thermal convection caused by application of the electric field, and can also act as a sieving medium, retarding the passage of molecules; gels can also simply serve to maintain the finished separation, so that a post electrophoresis stain can be applied [21].DNA Gel electrophoresis is usually performed for analytical purposes, often after amplification of DNA via PCR, but may be used as a preparative technique prior to use of other methods such as mass spectrometry (Fig.7), RFLP, PCR, cloning, DNA sequencing, or Southern blotting [22] for further characterization.

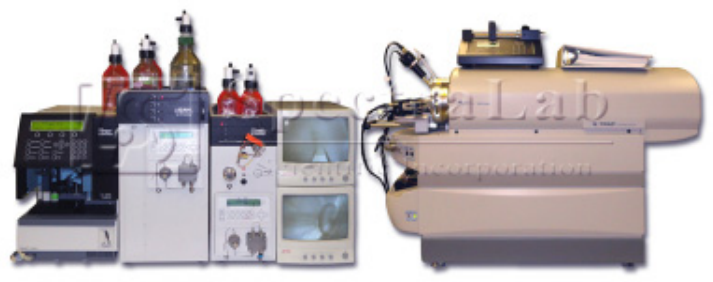

Figure 7. Mass spectrometry

\subsection{Hybridization methods}

In situ hybridization is a technique used to detect specific DNA and RNA sequences in a biological sample. Deoxyribonucleic acid (DNA) and ribonucleic acid (RNA) are macromolecules made up of different sequences of four nucleotide bases (adenine, guanine, uracil, cytosine, and thymidine). In situ hybridization takes advantage of the fact that each nucleotide base binds with a complementary nucleotide base. For instance, adenine binds with thymidine (in DNA) or uracil (in RNA) using hydrogen bonding. Similarly, guanine binds with cytosine.

In a specialized molecular biology laboratory, researchers can make a sequence of nucleotide bases that is complementary to a target sequence that occurs naturally in a cell (in a gene, for example). When this complementary sequence is exposed to the cell, it will bind with that naturally occuring target DNA or RNA in that cell, thus forming what is known as a hybrid. The complementary sequence thus can be used as a "probe" for cellular RNA or DNA.

Thus, the term "hybridization" refers to the chemical reaction between the probe and the DNA or RNA to be detected. If hybridization is performed on actual tissue sections, cells, or isolated chromosomes in order to detect the site where the DNA or RNA is located, it is said to be done "in situ." By contrast, "in vitro" hybridization takes place in a test tube or other apparatus, and is used to isolate DNA or RNA, or to determine sequence similarity of two nucleotide segments.

Application of the Probe for DNA or RNA to Tissues or Cells: In situ hybridization allows us to learn more about the geographical location of, for example, the messenger RNA (mRNA) 
in a cell or tissue. It can also tell us where a gene is located on a chromosome. Obviously, a detection system must be built into the technique to allow the cytochemist to visualize and map the geography of these molecules in the cells in question.

When in situ hybridization was first introduced, it was applied to isolated cell nuclei to detect specific DNA sequences. Early users applied the techniques to isolated chromosomal preparations in order to map the location of genes in those chromosomes. The technique has also been used to detect viral DNA in an infected cell. In situ hybridization of RNA has also been used to show that RNA synthesis (transcription) occurs in the nucleus, while protein synthesis (translation) occurs in the cytoplasm.

\subsection{Nucleic acid sequencing}

A process by which the sequence of nucleotides along a strand of DNA is determined. Originally a difficult process to carry out, DNA sequencing can now be done routinely by machines. The completion of the Human Genome Project in 2000 produced the largest DNA sequence ever assembled. To carry out the sequencing of the human genome, scientists cut the DNA up into short fragments, sequenced these fragments simultaneously, and then assembled the entire genome by using sophisticated computer techniques to match the fragments to each other.

\subsubsection{DNA microarrays}

Gene expression profiling using DNA microarrays holds great promise for the future of molecular diagnostics. This technology allows, in one assay, for simultaneous assessment of the expression rate of thousands of genes in a particular sample. The 2 types of DNA microarrays that are widely used are cDNA microarrays and oligonucleotide/ DNA chips.
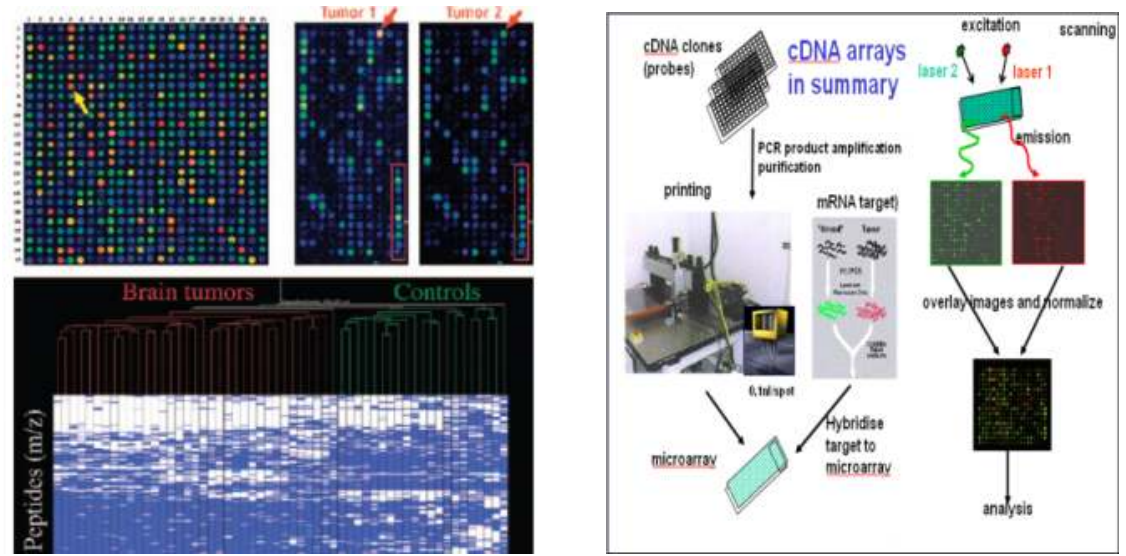

Figure 8. A lung primary tumor, for example, has a different fingerprint than an ovarian or colonic primary. This feature can be exploited in the setting of tumors of unknown primary, in which DNA microarrays have been shown to predict the correct primary site with an amazing accuracy rate of $99 \%$. 
In cDNA microarrays, DNA sequences complementary to a library of mRNA from thousands of genes are mechanically placed on a single glass slide. The immobilized cDNA sequences serve as anchoring probes to which mRNA extracted from the tested sample will specifically attach during hybridization. If the tested mRNA is first tagged with a fluorescent dye, the intensity of fluorescence at each anchoring probe location will be proportional to the amount of mRNA (degree of expression) of the gene at that location. A microarray reader displays the intensity of fluorescence at each cDNA location as a colored dot per gene location on a grid.

The applications of these technologies are limitless. By analyzing and comparing hundreds of tumor samples, databases of gene expression "fingerprints" are being built and specific patterns of expression linked to both prognosis and outcome of therapy.11-16 Patterns of gene expression in tumors are also linked to the primary site of origin (Fig.8).

\subsubsection{Proteomics}

The term proteomics indicates a large-scale characterization of the entire protein complement of a cell type, tissue, or organism. Two-dimensional gel electrophoresis has been, and remains, a widely used technique that offers a high-resolution capacity to separate and characterize proteins in complex mixtures. More recently, 2-dimensional electrophoresis (Fig.9) has been coupled with mass spectrometry analysis as a means to characterize complex milieus under study $[23,24]$. Several mass spectrometry platforms are commercially available. We will focus our discussion on surface-enhanced laser desorption/ionization technology [25, 26].

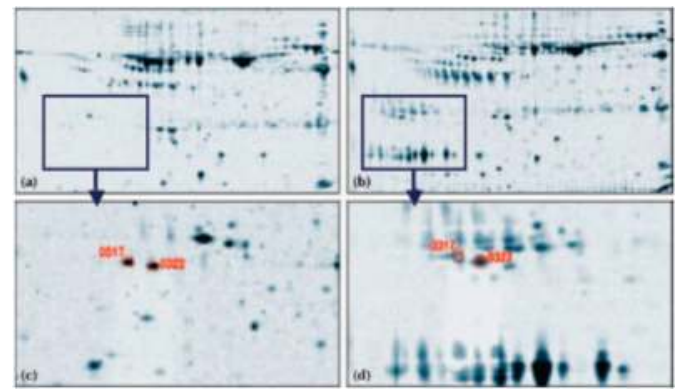

Figure 9. Proteomic analysis of nipple aspirate fluids using 2-dimensional gel electrophoresis. Spots in the bottom panel (enlarged from the top panel) indicate proteins expressed solely by nipple aspirate fluids from breasts containing tumor and not by the contralateral control breast. Copyright 2002 American Cancer Society. Reprinted with permission of Wiley-Liss, Inc, a subsidiary of John Wiley \& Sons, Inc. [27].

These technology uses matrix-assisted lasers desorption and time-of-flight analysis to allow fully automated separation of even minute amounts of protein components. The assay exploits variations in mass and electric charge of different protein components in a given sample (e.g., serum). It is the variation in time of flight-based on the mass/charge ratio of each peptide-that allows for separation of the different protein components. With the aid of computer analyses, results are displayed as peaks representing the different peptide components of a given sample. By characterizing proteomic "fingerprints" associated with a 
particular type of tumor, it is possible to identify an unknown sample that matches a previously defined fingerprint. This can be done on the basis of the "peaks" pattern without knowing which protein each peak represents. Appropriate integration of genomic and proteomic data is crucial to elucidate protein functions as they relate to pathogenesis. This integration will help highlight potential pathogenically important epigenetic deviations at the protein level.18 Integration of genomics and proteomics will no doubt facilitate discovery of novel drug target proteins and biomarkers of diseases.

\section{Molecular pathological diagnostics}

Molecular diagnostics can be widely defined as the measurement of deoxyribonucleic acid (DNA), ribonucleic acid (RNA), proteins, or other metabolites to detect certain genotypes, mutations, or biochemical changes that may be associated with certain states of health or disease, main applications of molecular genetic testing ... [28]. The emergence of molecular diagnostics is due to advances in biology that have resulted in an understanding of the mechanisms of normal and disease processes at the molecular level. Prior to this understanding, many disease states were diagnosed from morphologic observations.

The first widely used molecular tests were for infectious diseases, such as for hepatitis B and $\mathrm{C}$ or human papillomavirus (HPV), and this continues to be the largest molecular diagnostics market. Certain other fields, such as molecular tests for oncology, inherited conditions, cardiovascular disease, neurological disorders, and forensic testing, are rapidly growing areas of interest. Additionally, molecular diagnostics can be used to monitor a patient's response to a particular drug treatment.

Many different biological techniques fall under the "molecular diagnostics" umbrella. One of the most common techniques is the polymerase chain reaction (PCR), a method of producing large amounts of specifically defined DNA or RNA fragments that can then be used for multiple purposes, including pathogen identification and detection of aberrant gene expression associated with certain diseases. PCR fragments may also be sequenced to detect gene mutations connected to certain disease states, such as the detection of mutations in the BRCA1 and BRCA2 genes that are often associated with an increased risk of hereditary breast cancer and ovarian cancer.

\subsection{Molecular genetic testing}

Examples of main genetic testing..... [29].

- Bone Marrow Transplant Engraftment [Short tandem repeat (STR) polymorphic DNA markers used to distinguish patient from donor].

\subsubsection{Indications for molecular testing}

Allogeneic, HLA-matched hematopoietic stem cell transplantation (BMT) related or nonrelated donor. Donor and recipient specific DNA fragment patterns are utilized to distinguish the origins of each cell population. 


\subsection{Testing methodology}

Applied Biosystems AmpFLSTR Profiler Plus Kit utilizes short tandem repeat (STR) polymorphic DNA markers to distinguish patient and donor cells. Fluorescent multiplex polymerase chain reaction (PCR) is used to amplify the markers.

\subsubsection{Interpretation of DNA analysis}

DNA isolated from the WCB's (lymphoid or myeloid cells) of the patient and donor is characterized with 10 polymorphic DNA markers prior to the bone marrow transplantation. The patterns are compared and one marker is selected to distinguish donor and patient as uniquely different from one another.

This will be subsequently used to assess the status of donor cell engraftment. In related family members one or more of these markers may demonstrate high incidence of nonidentity in the BMT setting. The alleles range in size and heterozygosity.

In the general population, only identical twins have identical DNA profiles at all of these STR loci.When more than one of the ten STR markers has an informative (not identical) pattern between patient and donor, the one that demonstrates the most clearly distinguished differences in size or mobility is selected to follow engraftment status posttransplant. Amelogenin distinguishes $\mathrm{X}$ and $\mathrm{Y}$ chromosomes and may be used when the patient and donor are not of the same gender. Enriched subpopulations of hematopoietic cells (E.g. T lymphocytes and myeloid cells) may also be assayed

Specimen Requirements: Peripheral Blood--1 lavender-top (EDTA) tube. Invert several times to mix blood. Bone Marrow--Place 1-2 $\mathrm{mL}$ of anticoagulated bone marrow in a lavender-top (EDTA) tube. Invert several times to mix bone marrow. Do not freeze, forward promptly at ambient temperature. Buccal swab, cord blood, blood spots, and frozen tissue.

- $\quad$ BRCA Ashkenazi Jewish Mutations

BRCA1 and BRCA2 are human genes that belong to a class of genes known as tumor suppressors. Mutation of these genes has been linked to hereditary breast and ovarian cancer.

A woman's risk of developing breast and/or ovarian cancer is greatly increased if she inherits a deleterious (harmful) BRCA1 or BRCA2 mutation. Men with these mutations also have an increased risk of breast cancer. Both men and women who have harmful BRCA1 or BRCA2 mutations may be at increased risk of other cancers.

Genetic tests are available to check for BRCA1 and BRCA2 mutations (Fig.10). A blood sample is required for these tests, and genetic counseling is recommended before and after the tests. If a harmful BRCA1 or BRCA2 mutation is found, several options are available to help a person manage their cancer risk.

Several methods are available to test for $B R C A 1$ and $B R C A 2$ mutations. Most of these methods look for changes in BRCA1 and BRCA2 DNA. At least one method looks for changes in the proteins [ (PROH-teen) A] molecule made up of amino acids that are needed 
for the body to function properly. Proteins are the basis of body structures such as skin and hair and of substances such as enzymes, cytokines, and antibodies] produced by these genes. Frequently, a combination of methods is used.

A blood sample is needed for these tests. The blood is drawn in a laboratory, doctor's office, hospital, or clinic and then sent to a laboratory that specializes in the tests. It usually takes several weeks or longer to get the test results. Individuals who decide to get tested should check with their health care provider to find out when their test results might be available.
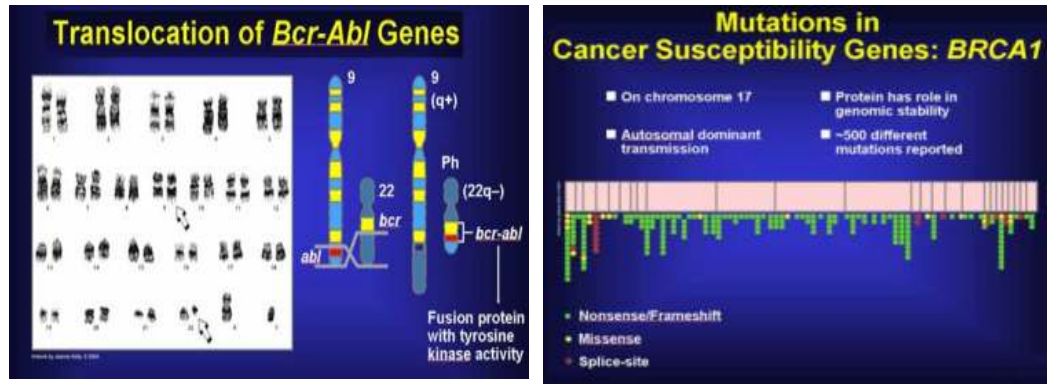

Figure 10. Genetic tests are to check for BRCA1 and BRCA2 mutations

\section{- $\quad$ Cystic Fibrosis Mutation Analysis}

Cystic fibrosis (CF) occurs at increased frequency in Caucasians and individuals of Ashkenazi Jewish descent, but can occur in any ethnic group (Fig.11). It is a disorder of mucus production, primarily affecting the pulmonary, gastrointestinal and reproductive systems. Although there is some variability of clinical expression, most individuals with CF require lifelong medical care and experience reduced life expectancy.

Molecular Testing: The preferred sample type is ACD or EDTA anticoagulated blood (pale yellow top or lavender top, $3 \mathrm{ml}$ ), which may be refrigerated up to 48 hours before analysis. A mutation panel is tested by PCR followed by oligonucleotide ligation assay using commercial analyte specific reagents (ASRs) available through Abbott diagnostics that were validated in laboratory. In diagnostic (as opposed to screening) tests, three polymorphisms that have the potential to confound mutation analysis are also evaluated along with analysis of the intron 8 polypyrimidine tract polymorphism (5T, 7T and 9T). Intron 8 analysis is also done as a reflex test when an $\mathrm{R} 117 \mathrm{H}$ mutation is detected because $5 \mathrm{~T}$ in cis with $\mathrm{R} 117 \mathrm{H}$ is a disease-related allele. Individuals of Ashkenazi Jewish descent are tested for D1152H in addition to the routine panel of mutations. Testing for $\mathrm{D} 1152 \mathrm{H}$ is performed by the Third Wave InPlexTM CF Assay. Results are reported as either consistent with a diagnosis of CF (two mutations found); at least carrier status (one mutation identified); or no detectable mutation (which reduces the probability of CF or carrier status). We recommend that screening (carrier testing) of the partner of a carrier be sent to an outside laboratory where more extensive mutation testing is possible. When screening is simultaneously requested on both partners, testing is done in-house. 


\section{- $\quad$ DNA Fingerprinting}

The process begins with a sample of an individual's DNA (typically called a "reference sample"). The most desirable method of collecting a reference sample is the use of a buccal swab, as this reduces the possibility of contamination. When this is not available (e.g. because a court order may be needed and not obtainable) other methods may need to be used to collect a sample of blood, saliva, semen, or other appropriate fluid or tissue from personal items (e.g. toothbrush, razor, etc.) or from stored samples (e.g. banked sperm or biopsy tissue). Samples obtained from blood relatives (biological relative) can provide an indication of an individual's profile, as could human remains which had been previously profiled.
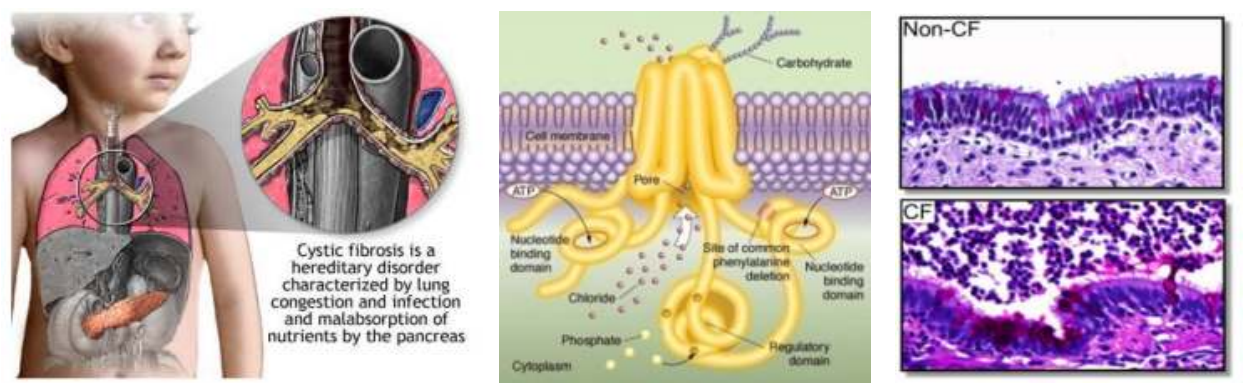

Figure 11. Cystic fibrosis (CF)

A reference sample is then analyzed to create the individual's DNA profile using one of a number of techniques, discussed below. The DNA profile is then compared against another sample to determine whether there is a genetic match.

Another technique, AmpFLP, or amplified fragment length polymorphism was also put into practice during the early 1990s. This technique was also faster than RFLP analysis and used PCR to amplify DNA samples. It relied on variable number tandem repeat (VNTR) polymorphisms to distinguish various alleles, which were separated on a polyacrylamide gel using an allelic ladder (as opposed to a molecular weight ladder). Bands could be visualized by silver staining the gel. One popular locus for fingerprinting was the D1S80 locus. As with all PCR based methods, highly degraded DNA or very small amounts of DNA may cause allelic dropout (causing a mistake in thinking a heterozygote is a homozygote) or other stochastic effects. In addition, because the analysis is done on a gel, very high number repeats may bunch together at the top of the gel, making it difficult to resolve. AmpFLP analysis can be highly automated, and allows for easy creation of phylogenetic trees based on comparing individual samples of DNA. Due to its relatively low cost and ease of set-up and operation, AmpFLP remains popular in lower income countries.

- Factor V Mutation Analysis

Factor V Leiden (F5) point mutation G1691A (Formerly FVL) (Fig.12). 
Indications for Molecular Testing. Family history of venous thrombosis- Unprovoked thrombotic event at $<45$ years of age- Women with multiple stillbirths or spontaneous abortions - Confirmation of diagnosis of F5 by non-molecular means

Testing Methodology. Screening utilizes polymerase chain reaction (PCR) and restriction fragment length polymorphism (RFLP) techniques to detect the Factor V Leiden point mutation (G1691A). The base pair change results in the loss of a recognition site for the restriction enzyme MnII. (PCR is utilized pursuant to a license agreement with Roche Molecular Systems, Inc.)

Interpretation of DNA analysis. Factor V Leiden, a point mutation (G1691A) in the gene coding for coagulation Factor $\mathrm{V}$, has been associated with an increased risk of venous thrombosis due to increased resistance to degradation of factor $\mathrm{V}$ by activated Protein $\mathrm{C}$. For individuals presenting with venous thrombosis, FVL occurs in $11-20 \%$ of those in all age groups and $50 \%$ of individuals under 50 years of age. Heterozygosity for this mutation produces a 7 - fold increase relative risk of venous thrombosis. Approximately $5 \%$ of Caucasians are heterozygous for this mutation. The homozygous occurrence of this mutation has been associated with an 80 - fold increased risk for venous thrombosis.

Specimen Requirements [Peripheral blood--1 lavender-top (EDTA) tube. Invert several times to mix blood].

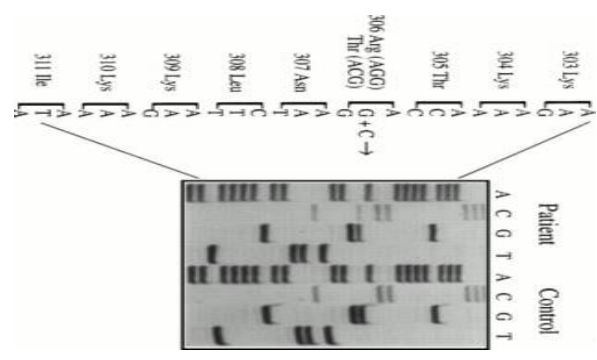

Figure 12. Factor V Leiden (F5) point mutation G1691A (Formerly FVL)

- Familial Mediterranean Fever Mutation

The MEFV gene (Fig.13) provides instructions for making a protein called pyrin (also known as marenostrin). Although pyrin's function is not fully understood, it likely assists in keeping the inflammation process under control. Inflammation occurs when the immune system sends signaling molecules and white blood cells to a site of injury or disease to fight microbial invaders and facilitate tissue repair. When this has been accomplished, the body stops the inflammatory response to prevent damage to its own cells and tissues.

Pyrin is produced in certain white blood cells (neutrophils, eosinophils, and monocytes) that play a role in inflammation and in fighting infection. Pyrin may direct the migration of white blood cells to sites of inflammation and stop or slow the inflammatory response when it is no longer needed. Pyrin also interacts with other molecules involved in fighting infection and in the inflammatory response. Research indicates that pyrin helps regulate 
inflammation by interacting with the cytoskeleton, the structural framework that helps to define the shape, size, and movement of a cell.
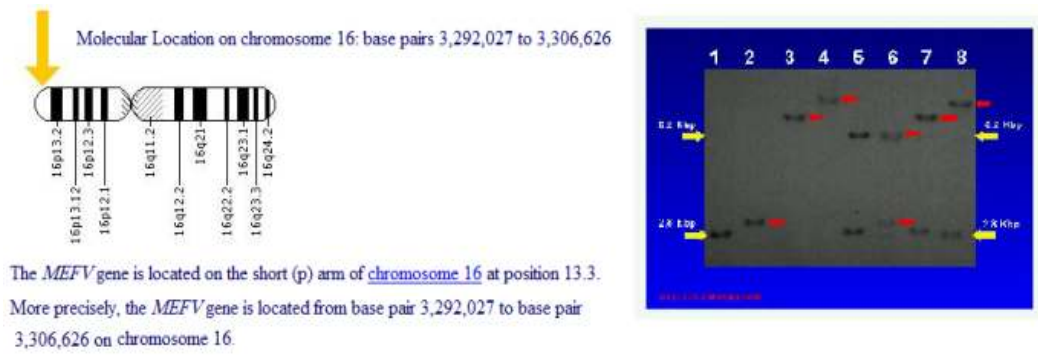

Figure 13. Familial Mediterranean Fever Mutation \& Fig.14: Fragile X syndrome (FXS), Martin-Bell syndrome.

- $\quad$ Fragile X Mutation Analysis

Fragile X syndrome (FXS), Martin-Bell syndrome, or Escalante's syndrome (more commonly used in South American countries,Fig.14), is a genetic syndrome that is the most common known single-gene cause of autism and the most common inherited cause of mental retardation among boys. It results in a spectrum of intellectual disability ranging from mild to severe as well as physical characteristics such as an elongated face, large or protruding ears, and larger testes (macroorchidism), behavioral characteristics such as stereotypical movements (e.g. hand-flapping), and social anxiety.

Fragile $X$ syndrome is associated with the expansion of the CGG trinucleotide repeat affecting the Fragile $X$ mental retardation 1 (FMR1) gene on the $X$ chromosome, resulting in a failure to express the fragile $X$ mental retardation protein (FMRP), which is required for normal neural development. Depending on the length of the CGG repeat, an allele may be classified as normal (unaffected by the syndrome), a premutation (at risk of fragile $\mathrm{X}$ associated disorders), or full mutation (usually affected by the syndrome). A definitive diagnosis of fragile $X$ syndrome is made through genetic testing to determine the number of CGG repeats. Testing for premutation carriers can also be carried out to allow for genetic counseling.

This molecular test detects the DNA expansion observed in approximately $99 \%$ of Fragile $X$ Syndrome carriers or affected individuals. Normal individuals have between about 6 and 50 FMR1 CGG repeats. The FMR1 CGG repeats tend to undergo expansion when repeat numbers exceed about 50. Premutation carrier females and normal transmitting males have between about 50 and 200 repeats. Affected males typically have many more than 200 repeats. Females with an X chromosome having greater than 200 FMR1 CGG repeats may or may not be affected depending on X chromosome inactivation patterns. FMR1 CGG repeats of about 50 to 200 show a dynamic instability directly related to their length, to the sex of the transmitting individual, and to subtle DNA sequence variation within the repeat itself. Premutation alleles tend to be unstable when transmitted by females and stable when transmitted by males (stability in males may be a consequence of selection against expanded FMR1 CGG alleles during spermatogenesis). Repeats greater than about 90-100 repeats have 
nearly a $100 \%$ risk of expansion into the affected range when transmitted by a female. The FMR1 CGG repeat demonstrates significant somatic instability when repeat sizes enter the premutation range. Premutation and larger sized CGG repeats determined from peripheral blood DNA may not reflect FMR1 CGG repeat sizes in other tissues.

- Friedreich's Ataxia Mutation Analysis

Friedreich's ataxia is an inherited disease that causes progressive damage to the nervous system, resulting in symptoms ranging from gait disturbance to speech problems; it can also lead to heart disease and diabetes (Fig.15).
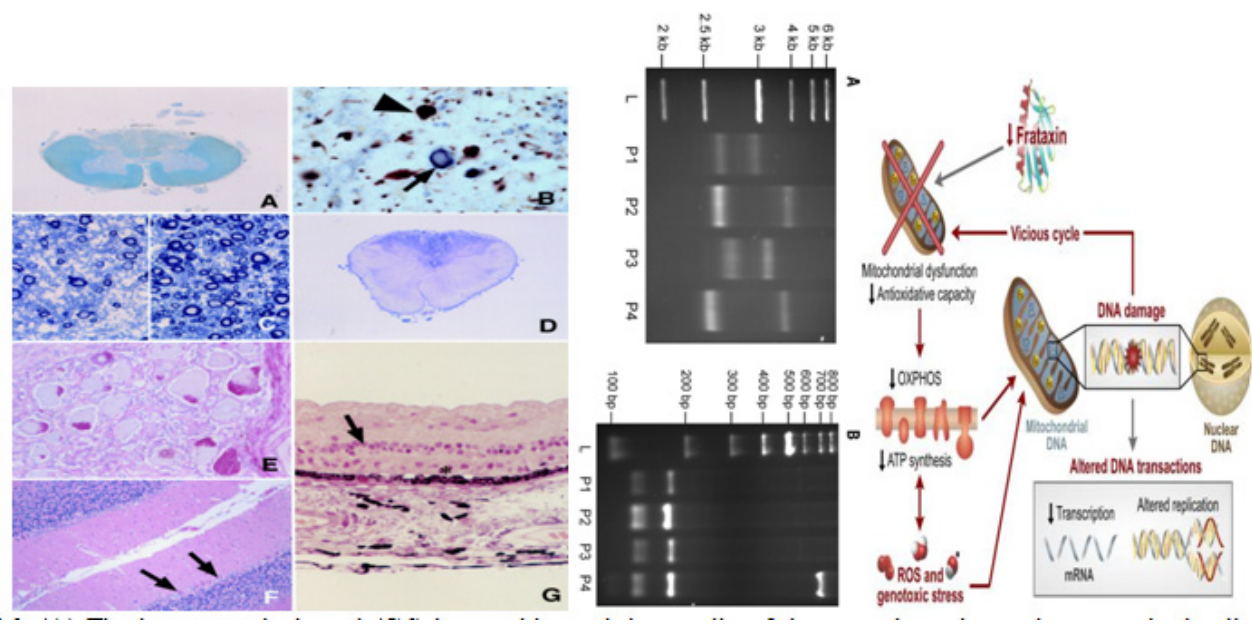

Figure 14. (A) The lower cervical cord (C6) is atrophic, and the myelin of the posterior column shows marked pallor (Klüver-Barrera stains originally×6.0). (B) Numerous axonal spheroids immunostained by antineurofilament antibody, SMI-31 (brown, arrow head) and corpora amylacea by antibiquitin antibody (purple, arrow) in the posterior column nucleus (originally $\times 514$ ). (C) The gracile fasciculi of the upper cervical cord (C3, left) and lower lumbar cord (L2, right) (toluidine blue stain $\times 514)$, show decreased axon density and thin myelin. These changes are more marked in C3 than those in L2. (D) Lower medulla (Holzer stain originally $\times 4.4$ ). Marked gliosis is present in the gracile and cuneate nuclei. (E) The dorsal root ganglion of right C7 (periodic acid-Shiff originally $\times 210$ ). Ganglion cells are well preserved and Nageotte's nodules are very rare. (F) Cerebellar hemisphere (haematoxylin and eosin originally×64). There is focal loss of Purkinje cells. Purkinje cells have almost disappeared in the upper folium, but are relatively preserved in the lower folium (arrow). (G) Retina (haematoxylin and eosin originally $\times 257$ ). The outer segment layer of photoreceptor cells has disappeared (asterisk) and the outer nuclear layer is not obvious. The inner layers of the retina are thin and atrophic (arrow). Journal of Neurology, Neurosurgery \& Psychiatryjnnp.bmj.com- J Neurol Neurosurg Psychiatry 2000;68:521-525 doi:10.1136/jnnp.68.4.521. Analysis of the GAA trinucleotide repeats expansion in the FXN gene of individuals with Friedreich ataxia. (A) The length of the GAA repeat region in each allele of the FXN gene was determined by long-range PCR and agarose gel electrophoresis. Patient 1, 770 and 870 repeats; Patient 2, 760 and 1170 repeats; Patient 3, 790 and 940 repeats; Patient 4, 650 and 1140 repeats; L: DNA size ladder. (B) PCR products following digestion with MboII. Complete digestion of pure GAA repeat sequences leaves flanking regions of 157 bp and 125 bp. The occurrence of an additional band of $\sim 680 \mathrm{bp}$ in lane P4 indicates the presence of non-GAA sequence within the GAA trinucleotide repeat expansion region [31]. 
The ataxia of Friedreich's ataxia results from the degeneration of nerve tissue in the spinal cord, in particular sensory neurons essential (through connections with the cerebellum) for directing muscle movement of the arms and legs. The spinal cord becomes thinner and nerve cells lose some of their myelin sheath (the insulating covering on some nerve cells that helps conduct nerve impulses).

The condition is named after the German physician Nikolaus Friedreich, who first described it in the 1860s [30].

- Hereditary Hemochromatosis

Hemochromatosis gene (HFE) testing is a blood test used to check for hereditary hemochromatosis, an inherited disorder that causes the body to absorb too much iron. The iron then builds up in the blood, liver, heart, pancreas, joints, skin, and other organs (Fig.16).

In its early stages, hemochromatosis can cause joint and belly pain, weakness, lack of energy, and weight loss. It can also cause scarring of the liver (cirrhosis), darkening of the skin, diabetes, infertility, heart failure, irregular heartbeats (arrhythmia), and arthritis. But many people do not have symptoms in the early stages.
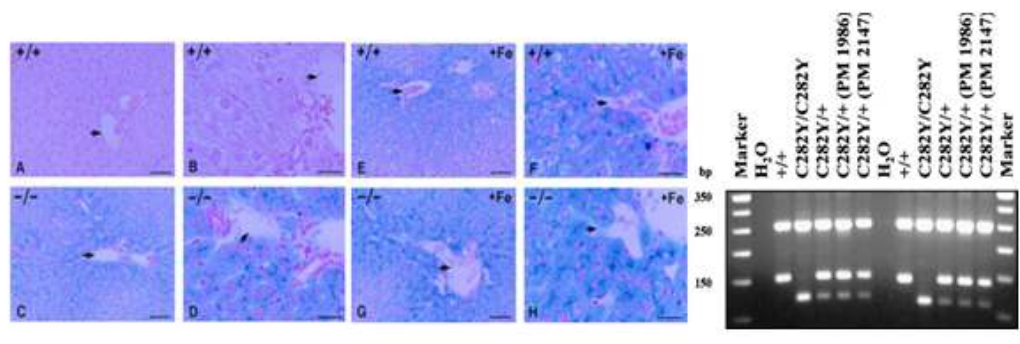

Figure 15. Perls' Prussian blue staining of liver sections from $\mathrm{HFE}^{+/+}$and $\mathrm{HFE}^{-/-}$mice fed control diet $(A-D)$ or control diet supplemented with $2 \%(\mathrm{wt} / \mathrm{wt})$ carbonyl iron $(E-H)$. Shown are low-power views $(A, C, E$, and $G)$ and high-power views $(B, D, F$, and $H)$ of sections. $A$ and $B$ show the absence of stainable iron in the $+/-$ mouse liver fed the control diet. $C$ and $D$ show prominent stainable iron in hepatocytes with periportal predominance in liver from $\mathrm{HFE}^{-/-}$mice fed the control diet. $E$ and $F$ show iron accumulation in $\mathrm{HFE}^{+/+}$mouse liver in response to iron loading. $G$ and $H$ show the stainable iron in the $\mathrm{HFE}^{-/-}$mice after 2 weeks of feeding with the iron-supplemented diet. The arrows indicate the location of branches of the portal vein. (Bars: $A, C, E$, and $G=50 \mu \mathrm{m}$ in the low-power views; $B, D, F$, and $H=20 \mu \mathrm{m}$ in the high-power views.)

In men, hereditary hemochromatosis is usually found between the ages of 40 and 60 . In women, it is not usually found until after menopause because, until that time, women regularly lose blood and iron during their monthly periods. Genetic testing for hemochromatosis involves a simple blood test. DNA from the blood is extracted and the HFE gene is tested for two specific mutations in the gene known to cause the disease in most people. There are two laboratory techniques typically used to detect the two mutations: allele-specific oligonucleotide hybridization (ASO) and restriction fragment length polymorphism (RFLP) analyses. Both of these tests are designed to detect whether a specific 
mutation is present or absent in a DNA sample. These tests will also determine if an individual is a carrier of either mutation. The two mutations typically tested for are most commonly called C282Y and H63D. The mutations are named based on their location within the HFE gene and the change that they cause in the gene's instructions. The two mutations differ in how frequently they are found in the population, and in how severe your hemochromatosis is if you have them. There are other rare mutations that may predispose individuals towards abnormal iron storage. However they are not yet part of most laboratories testing panels for hemochromatosis.

- Huntington Disease

Huntington's disease (HD) is a neurodegenerative genetic disorder that affects muscle coordination and leads to cognitive decline and psychiatric problems (Fig.17). It typically becomes noticeable in mid-adult life. HD is the most common genetic cause of abnormal involuntary writhing movements called chorea, and indeed the disease used to be called Huntington's chorea.

Because HD follows an autosomal dominant pattern of inheritance, there is a strong motivation for individuals who are at risk of inheriting it to seek a diagnosis. The genetic test for HD consists of a blood test which counts the numbers of CAG repeats in each of the HTT alleles [32]. A positive result is not considered a diagnosis, since it may be obtained decades before the symptoms begin. However, a negative test means that the individual does not carry the expanded copy of the gene and will not develop HD [33].
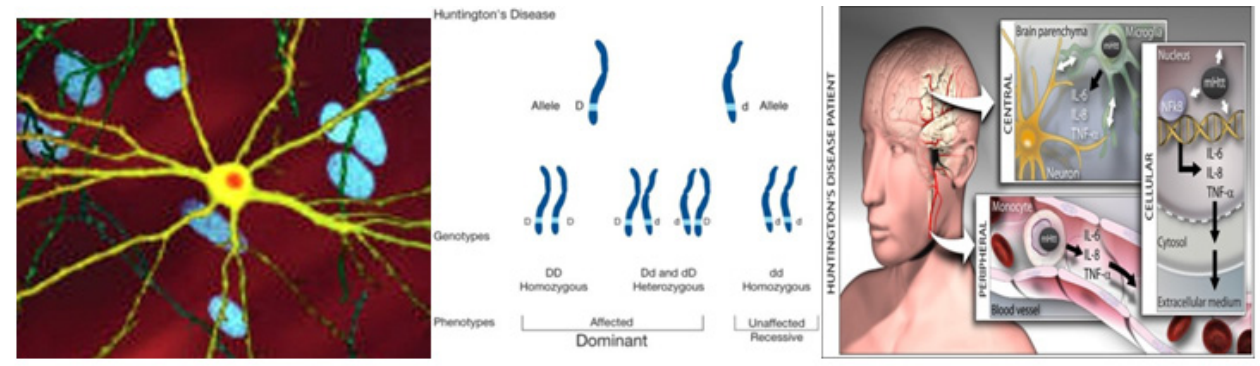

Figure 16. Huntington's disease (HD) is a neurodegenerative genetic disorder that affects muscle coordination and leads to cognitive decline and psychiatric problems. Neuron with inclusion (stained orange) caused by HD, image width $250 \mu \mathrm{m}$.

A pre-symptomatic test is a life-changing event and a very personal decision [33]. The main reason given for choosing testing for HD is to aid in career and family decisions [33]. Over 95\% of individuals at risk of inheriting HD do not proceed with testing, mostly because there is no treatment. [ ${ }^{38]} \mathrm{A}$ key issue is the anxiety an individual experiences about not knowing whether they will eventually develop HD, compared to the impact of a positive result [33] . Irrespective of the result, stress levels have been found to be lower two years after being tested, but the risk of suicide is increased after a positive test result [33] . Individuals found to have not inherited the disorder may experience survivor guilt with regard to family members who are affected [12]. Other factors taken into account when 
considering testing include the possibility of discrimination and the implications of a positive result, which usually means a parent has an affected gene and that the individual's siblings will be at risk of inheriting it. Genetic counseling in HD can provide information, advice and support for initial decision-making, and then, if chosen, throughout all stages of the testing process. ${ }^{[39]}$ Counseling and guidelines on the use of genetic testing for HD have become models for other genetic disorders, such as autosomal dominant cerebellar ataxias [38]. Presymptomatic testing for HD has also influenced testing for other illnesses with genetic variants such as polycystic kidney disease, familial Alzheimer's disease and breast cancer [34].

\section{Molecular oncology testing}

\section{- B-Cell Gene Rearrangement}

The B- and T-cell rearrangement test [35] can detect a monoclonal population of B- and Tcells, strongly indicative of neoplasia. This is accomplished through the use of DNA probes. The presence of a unique band on the Southern blot (Fig.18) signifies a monoclonal gene rearrangement, which can make or confirm a diagnosis of a lymphoproliferative disorder and classify the lineage as B- or T-cell. T-cell neoplasms generally behave more aggressively than B-cell neoplasms, which can have important implications for prognosis and therapy. The unique gene rearrangement "signature" can be followed during and after therapy to document remission or recurrence. The most commonly examined samples are from the blood, bone marrow, and lymph nodes, but any tissue or fluid suspected of harboring a lymphoid neoplasm can be examined.

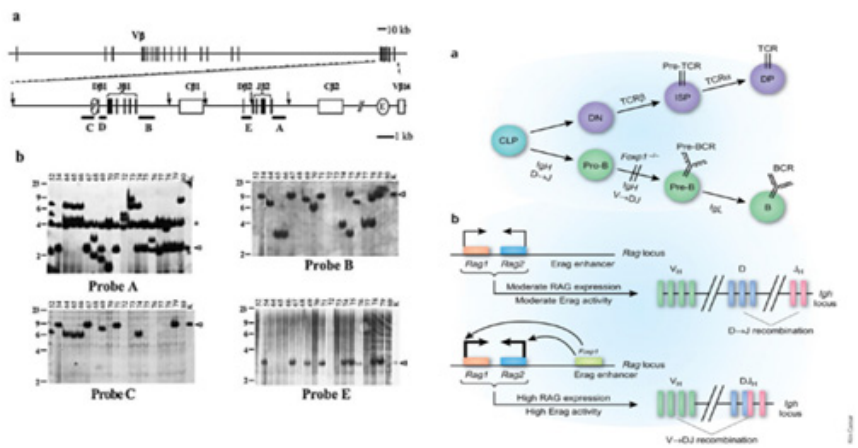

Figure 17. B-Lymphocyte Immunoglobulin; Heavy Chain Gene Rearrangement. Detection of monoclonal B-cell populations in B-lymph proliferative disorders.

This test is indicated for the accurate differentiation of malignant vs. benign lymphoproliferative disorders and for establishing $\mathrm{T}$ tumor cell lineage. Clonal proliferations of $\mathrm{T}$ lymphocytes can be detected by the identification of specific DNA rearrangements in the T-cell gamma chain antigen receptor gene. The majority of lymphocytic leukemias and non-Hodgkin's lymphomas arise from a clonal proliferation of a single lymphoid cell that has become aberrant. T cells normally differentiate from stem 
(precursor) cells in a highly specific and sequential manner. The malignant transformation may take place at any stage in the maturation process and when it occurs, the resulting malignant clone bears the characteristics of the originally transformed cell and is called monoclonal. Some of the early events in the maturation process of lymphoid cells involve specific nucleic acid rearrangements within the gamma chain antigen receptor gene in T-cells.

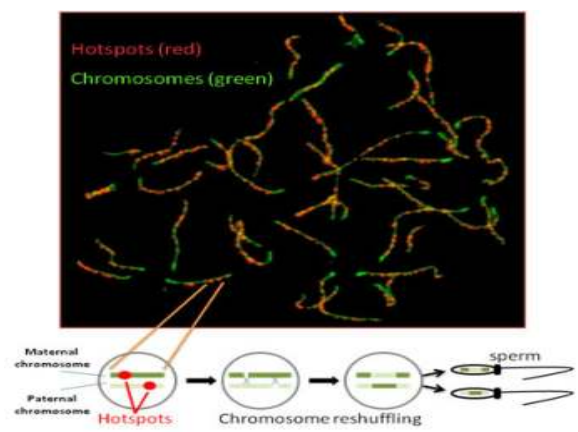

Figure 18. In this image, hundredfold magnification of a single sperm precursor cell shows the chromosomes - in green - and the places where these chromosomes are most likely to break apart and re-form, called genetic recombination hotspots - in red. Genetic rearrangements at these hotspots have the potential to shuffle maternal and paternal chromosomes, the end results of which ensure that the genetic information in every sperm cell is unique. Source: Fatima Smagulova, Ph.D., USU, and Kevin Brick, Ph.D., NIDDK, NIH.

This test is indicated for the accurate differentiation of malignant vs. benign lymphoproliferative disorders and for establishing B tumor cell lineage. Clonal proliferations of B-lymphocytes can be detected by the identification of specific DNA rearrangements in the immunoglobulin gene. The majority of lymphocytic leukemias and non-Hodgkin's lymphomas arise from a clonal proliferation of a single lymphoid cell that has become aberrant. B cells normally differentiate from stem (precursor) cells in a highly specific and sequential manner. The malignant transformation may take place at any stage in the maturation process and when it occurs the resulting malignant clone bears the characteristics of the originally transformed cell and is called monoclonal. Some of the early events in the maturation process of lymphoid cells involve specific nucleic acid rearrangements within the immunoglobulin genes in B cells. To detect B cell gene rearrangements using PCR, primers are constructed to amplify the DNA between the conserved framework (FR) and joining (J) regions. These conserved regions lie on either side of an area within the V-J region where programmed genetic rearrangements occur during maturation. In the germline configuration found in somatic cells, these areas are widely separated (by more than 70KB) making PCR amplification across the area impossible. The sequence alteration brought about by gene rearrangement brings these regions into close proximity, making the area of amplifiable length. Each B cell has a single productive V-J rearrangement that is unique in both length and sequence. The template-free incorporation of nucleotides (N-regions) between the spliced V, D, and J gene segments also adds uniqueness to the PCR product. Therefore, when this region is amplified using DNA 
primers that flank this region, a clonal population of cells yields one or two prominent amplified products (amplicons) within the expected size ranges. Two products are produced in cases when the initial rearrangement was non-productive and was followed by rearrangement of the other homologous chromosome. In contrast, DNA from a normal or polyclonal population produces a bell-shaped curve (or Gaussian distribution) of amplicon products that reflects the heterogeneous population of V-J region rearrangements. Specimen: Whole blood, bone marrow or tissue( $10 \mathrm{~mL}$ blood; $4 \mathrm{~mL}$ bone marrow; $0.2 \mathrm{~g}$ or more of tissue)

- $\quad$ BCL-2 Gene Rearrangement

Gene Rearrangement bcl-2; Major Breakpoint Region (MBR); minor cluster region (mcr); $\mathrm{t}(14: 18)$ Translocation. Applies to Follicular B-Cell Lymphomas. A translocation between immunoglobulin genes (heavy chain or light chain genes) and $b c l-2$ results in the over expression of $b c l-2$ protein and thus the expansion of B cells due to inhibition of cell death. This type of translocation is found in $100 \%$ at small cleaved type, $76 \%$ to $85 \%$ at mixed cell and $59 \%$ to $75 \%$ at large cell types of Bcell lymphoma. It is found in some cases of chronic lymphocytic leukemia, acute lymphoblastic leukemia, and small noncleaved cell lymphoma as well as some cases of Hodgkin's lymphoma and myeloid neoplasms. The $t(14 ; 18)$ is rarely detected in monocytoid B-cell lymphoma and MALT lymphomas. $b c l-2$ rearrangement is not pathognomonic for lymphomas. It is found in $10 \%$ of reactive lymph nodes, and in some normal cells (eg, lymphoid and myeloid precursors, medullary thymocytes, $\mathrm{T}$ cells, nongerminal center B cells, and plasma cells). It is not expressed in centers.

- $\quad$ BCR-ABL Gene Rearrangement

Translocation bcr/abl; Gene Rearrangement bcr; Philadelphia Chromosome; t (9:22) Applies to Acute Myelogenous Leukemia (ALL); Chronic Myelogenous Leukemia (CML)

- $\quad$ BRAF Gene Mutation Detection

BRAF is downstream of KRAS in a signaling pathway involved in cell cycling. Both KRAS and BRAF are prone to mutations in sporadic colorectal carcinomas (CRC).

This assay is capable of detecting the V600E mutation in exon 15 of BRAF. The much rarer V600A or V600G mutations would also be detectable. Detection includes PCR amplification and a single-base extension reaction that generates allele-specific fluorescently labeled probes, detectable by capillary electrophoresis.

Several studies have demonstrated that mutations in KRAS lead to constitutive activation of this pathway, which may lead to cancer progression, and this activation results in a failure to respond to anti-EGFR therapy. Recent published results indicate that mutations in the KRAS gene are present in approximately $40 \%$ of patients with metastatic colorectal adenocarcinoma, and the presence of a K-ras mutation isassociated with resistance to cetuximab and panitumumab. BRAF mutations are responsible for an additional $12-15 \%$ of patients who fail to respond to anti-EGFR treatment. This finding suggests that testing for the BRAF V600E mutation can compliment KRAS mutation analysis and may be as important as KRAS testing for treatment decisions. 
- c-kit Mutation Detection for Systemic Mastocytosis

Nucleotide substitutions at and adjacent to codon 816 in the catalytic domain of c-kit are common in SM. Detection of a codon 816 c-kit mutation is included as a minor diagnostic criterion in the WHO's diagnostic criteria for SM. Determining mutational status of the c-kit gene also has pharmacogenetic implications in patients considered for investigational mast cell cytoreductive therapies and targeted small-molecule tyrosine kinase inhibitors Sequencing analysis. Polymerase Chain Reaction (PCR) is performed for DNA amplification. Primers are designed specifically for exon 17 of the c-kit gene. PCR products are sequenced and analyzed on the ABI 3130xl Genetic Analyzer.

\section{- JAK2 Mutation Analysis}

The JAK2 V617F substitution, located in the pseudokinase domain of JAK2, relieves the autoinhibition of its kinase activity; the resulting constitutively active kinase augments downstream JAK2-STAT signaling pathways. Other JAK2 mutations in humans include translocations, point mutations, deletions, and insertions [36, 37]. However, the most frequent mutations are those seen in patients with JAK2 V617F-negative polycythemia vera or idiopathic erythrocytosis (Fig.20 and 21), the exon 12 mutations. Documented highfrequency JAK2 exon 12 mutations include in-frame deletions, missense, and tandem point mutations such as del/F537-K539ins/L, del/N542-E543, K539L, and H538QK539L [38]. Whereas JAK2 V617F mutations are typically homozygous (by mitotic recombination), exon 12 mutations are often heterozygous in patients with polycythemia vera. In addition, exon 12 mutations can induce cytokine-independent hypersensitive proliferation in erythropoietin-expressing cell lines and are sufficient for the development of a polycythemia vera-like phenotype in a murine model.

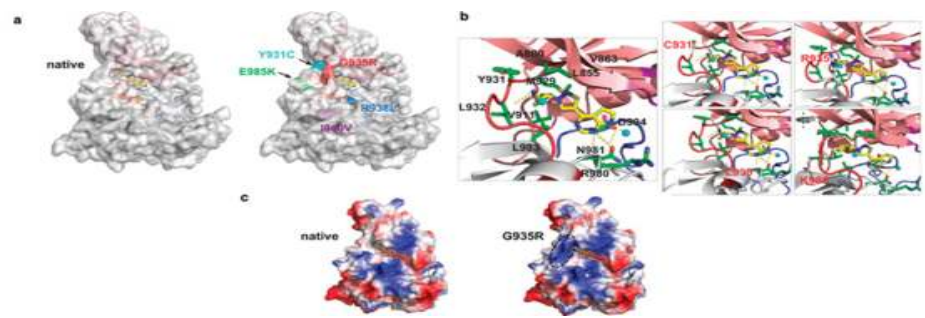

Figure 19. Structural analysis of JAK2V617F kinase domain mutations. (a) Cartoon and transparent surface representation of ruxolitinib-docked JAK2 kinase domain $(\mathbf{a}, \mathbf{b})$ (left) and JAK2 with location of point mutations that lead to drug resistance (right). N-terminal lobe (salmon), C-terminal lobe (gray), glycine loop (purple), activation loop (blue) and hinge region (red) form the boundaries for the binding site of ruxolitinib (stick representation in yellow (carbon) and blue (nitrogen)). The I960V side chain (purple) is buried within the protein interior. (b) Enlarged ruxolitinib-binding pocket with secondary structure elements (cartoon) and the interactions of the side chains (labeled sticks) with the inhibitor. Hydrogen bonds between the inhibitor and the protein are indicated as dotted yellow lines (one hydrogen bond between backbone of Y931 and L932; and two hydrogen bonds with R980 and N981 and pyrrolopyrimidine ring of the inhibitor; additional hydrogen bonds are with water molecules (cyan spheres) . Mutated amino acids are labeled red (right panels). (c) Surface electrostatic potential representation of the native (left) and G935R (right) containing JAK2 JH1 domain with ruxolitinib. Charged surfaces are displayed in shades of blue (positive), red (negative) and white (non-polar) [39]. 


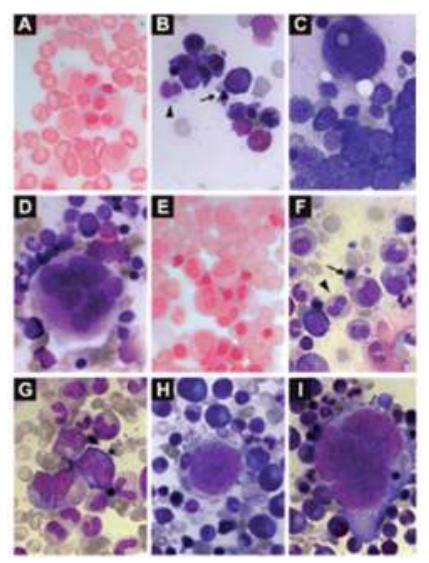

Figure 20. Bone marrow morphology demonstrating both dysplastic and proliferative features in a JAK V617F negative patient (n. 511; A-D) and a patient with the mutation (n. 510; E-I). Ringed sideroblastosis $(\mathrm{A}, \mathrm{E})$ associated with immaturity, megaloblastoid changes and abnormal nuclear budding (arrows) and binuclearity (asterix) of erythroblasts (B,C,F,G). Dysgranulopoiesis with numerous hypogranular (arrowheads) myeloid cells (B,F,G; Pappenheim's stain). Evidence of both small megakaryocytes with round nuclei and mature cytoplasm $(\mathrm{C}, \mathrm{H})$ and large multinucleated forms (D, I). A, E, Perls' stain; B-D, F-I, Pappenheim's stain; $\times 1000$.

\section{- $\quad$ KRAS Gene Mutation Detection}

Mutations in the KRAS oncogene are highly prevalent in human tumors, and they most commonly occur in codons 12,13 , and 61 . K-ras mutations have been detected in pancreatic, colorectal, lung, endometrial, gallbladder, and thyroid cancer at variable frequency. Accumulating evidence indicates that these mutations may play a role in prognosis and drug response. Specifically, recent published results indicate that mutations in the KRAS gene are present in approximately $40 \%$ of patients with metastatic colorectal adenocarcinoma, and the presence of a K-ras mutation is associated with resistance to cetuximab and panitumumab.

The ViennaLab K-ras StripAssay kit will detect 10 KRAS gene mutations in codons 12 and 13: G12V, G12D, G12L, G12S, G12A, G12I, G12C, G12R, G13C and G13D.

Specimen Formalin-fixed paraffin-embedded (FFPE) tissue, 10 precut, unstained slides from paraffin block in $10 \mu \mathrm{m}$ (10-micron) sections or formalin-fixed paraffin-embedded tissue block containing $\geq 50 \%$ tumor. Either option needs to be accompanied by an H\&E reference slide. Detection includes PCR amplification using biotinylated primers, reverse hybridization of PCR products to test strips containing specific mutant oligonucleotide probes, and visualization of bound products with streptavidin-alkaline phosphatase and color substrates.

- Microsatellite Instability

Microsatellites, also known as Simple Sequence Repeats (SSRs) or short tandem repeats (STRs), are repeating sequences of 2-6 base pairs of DNA. 
Microsatellites are typically co-dominant. They are used as molecular markers in genetics, for kinship, population and other studies. They can also be used to study gene duplication or deletion. Microsatellites are also known to be causative agents in human disease, especially neurodegenerative disorders and cancer.

In cells with mutations in DNA repair genes, however, some of these sequences accumulate errors and become longer or shorter. The appearance of abnormally long or short microsatellites in an individual's DNA is referred to as microsatellite instability. Microsatellite instability (MSI) is a condition manifested by damaged DNA due to defects in the normal DNA repair process. Sections of DNA called microsatellites, which consist of a sequence of repeating units of 1-6 base pairs in length, become unstable and can shorten or lengthen. Microsatellites are also known as simple sequence repeats (SSRs). Specimen,1 normal tissue and 1 tumor tissue. Normal tissue may be substituted by a sample blood Each tissue sample must be $\geq 25 \mathrm{mg}$.. Amplification by Polymerase Chain Reaction (PCR) of 5 microsatellite short tandem repeats and detection of these alleles by electrophoresis and sizing on an automated fluorescence detector.

Muir Torre syndrome [MTS] is a rare autosomal dominant inherited genodermatosis with malignant potential. It consists of at least one sebaceous gland tumor such as sebaceous adenoma, epithelioma, or carcinoma, with at least one visceral malignancy [40].

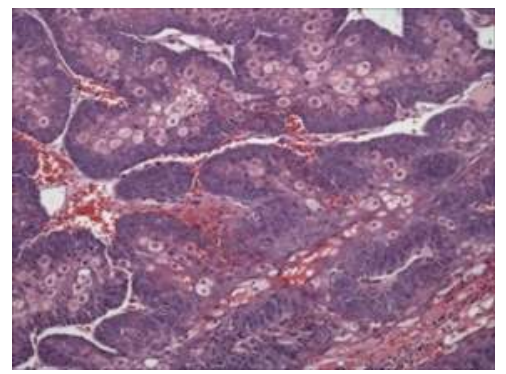

Figure 21. Components of Muir-Torre Syndrome. a). Sebaceous adenoma (100X) b). Colonic mucinous adenocarcinoma (100X).

Genetic mutations have been identified as the cause of inherited cancer risk in some colon cancer-prone families. The most common clinical syndromes associated with these mutations include familial adenomatous polyposis (FAP) and hereditary non-polyposis colorectal cancer (HNPCC). The former is associated with mutations of the APC gene, and the latter with mutations of MLH1, MSH2, MSH6, and PMS2 genes [41]. These inherited syndromes are estimated to account for only $2 \%$ to $6 \%$ of colorectal cancer cases overall. Turcot syndrome is a clinically defined, inherited syndrome associated with both colorectal cancer and a primary brain tumor.

- PMLRAR Alpha $\mathrm{t}(15 ; 17)$ Translocation

AMLs (Acute Myeloid Leukemias, Fig.23) [42] are characterized with chromosomal translocations resulting in the formation of fusion proteins. Understanding PML (Acute 
Promyelocytic Leukemia Inducer) function has become an area of intense research because of its involvement in the pathogenesis of APL (Acute Promyelocytic Leukemia), a distinct subtype of Myeloid Leukemia. In the vast majority of APL case studies, the PML gene (on Chromosome-15) fuses to the RAR-Alpha gene (Retinoic Acid Receptor-Alpha) (on Chromosome-17) as a consequence of reciprocal and balanced chromosomal translocations. In the $t(15 ; 17)$ chromosomal translocation, which is specific for APL, PML is found in a reciprocal translocation with the RAR-Alpha resulting in the formation of PML-RAR-Alpha and RAR-Alpha-PML fusion proteins . In a normal cell Vitamin-A (all-trans-Retinol) from dietary sources is converted to all-trans-Retinoic Acid in the liver through Retinol Metabolism and all-trans-Retinoic Acid is translocated to tissues by CRABP (Cellular Retinoic Acid Binding Protein) where it regulates target genes by binding with RARs (Retinoic Acid Receptors). Retinoic Acid is the only metabolite of Vitamin-A which regulates gene expression and all-trans-Retinoic Acid, the Carboxylic Acid form of Vitamin-A is of biological significance since it has high circulating levels than other isomers of Retinoic Acid. Biologically active ligands for the RARs include all-trans-Retinoic Acid, 9-cis-Retinoic Acid among others. 13-cis-Retinoic Acid is not a ligand for the RARs, but, it is readily converted into a RAR ligand by intracellular reciprocal isomerization. Less is understood about the fate of intracellular all-trans-Retinoic Acid is its isomerization to 9-cis-Retinoic Acid and 13-cis-Retinoic Acid. The RARs have a conserved modular structure consisting of six regions from A-F, viz., AF-1 or A/B (Amino-Terminal Activating Factor-1 Transcriptional Activation) Domain; a zinc-finger DBD or C (DNA-Binding Domain); a CoR or D (Hinge/Corepressor Binding) Domain; a LBD or AF-2 or E (Ligand-Binding/Transcriptional Activation) Domain; and a variable F (Carboxyl-Terminal) Domain. In RAR the DBD binds to the RARE (Retinoic Acid Response Element) region in the DNA. The RAREs consists of DRs (Direct Repeats) of AGG/TTCA motif with a spacer region of (n)25. Upon Retinoic Acid binding, RAR-Alpha regulates Retinoic Acid mediated gene expression and transactivates PML target genes critical for the induction of Myeloid Hemopoietic Cells' terminal differentiation.

In this assay, extracted RNA is subjected to 2 separate quantitative real-time reverse transcription-polymerase chain reaction (RT-PCR) procedures to detect the 2 types of PML/RARA fusion transcripts (long and short isoforms). An additional amplification for the abl gene is performed as a control for sample RNA quality and as a reference for relative quantification. The results are reported as positive or negative; the ratio of target (PML/RARA) to control (ABL) mRNA is reported for positive specimens. The isoform (short or long) is also reported. If available, a previously stored sample will be tested alongside the current specimen to assess quantitative changes with time (trend). The analytical sensitivity of this test is 1 tumor cell in 100,000 normal cells.

In APL cells due to $t(15 ; 17)$ chromosomal translocation the fusion protein PML-RAR-Alpha retains both DBD and LBD of RAR-Alpha, compete with normal RAR-Alpha for ligand binding and inhibits its transcriptional function through aberrant recruitment of HDACs (Histone Deacetylases). Recruitment of HDACs to PML also leads to inhibition of p53 activity and Sumolation. HDACs therefore, represent an ideal candidate for blocking the 
action of the fusion proteins. PML-RAR-Alpha and RAR-Alpha-PML fusion protein expression disrupts formation of NBs and paralyzes Tumor Suppression, Cellular Senescence, Mature PML degradation and normal Cell Growth and Survival. Co-expression of RAR-Alpha-PML with PML-RAR-Alpha thus results in an increase of Leukemia incidence and makes a cell more prone to pathogen invasions. All-trans-Retinoic Acid is a standard therapy for the management of APL. However, 13-cis-Retinoic Acid and 9-cisRetinoic Acid implication reduce the incidence of secondary head and neck tumors and APL, respectively. It is apparent that PML is essential for critical tumor suppressive pathways that are deregulated in APL and therapies such as induction of Retinoic Acid and As2O3 can be helpful in restoring normal PML function in APL cells that can cause the reappearance of NBs, and the reversal of T-Cell Gene Rearrangement.


Figure 22. Under normal conditions PML is a potential Tumor Suppressor and is involved in Cellular Senescence, a process that controls Oncogenic Signaling leading to normal Cell Growth and Survival. PML is the organizer of nuclear matrix domains, NBs (Nuclear Bodies), with a proposed role in Apoptosis control. PML being a member of the RBCC (RING-B-Box-Coiled-Coil) Protein Family, contains three Zinc Finger-Like domains (a RING Finger and two B-Boxes) and a Coiled-Coil Dimerization domain. PML organizes NBs by targeting proteins such as Sp100 (Nuclear AntigenSp100), p53, Rb (Retinoblastoma) or Daxx onto these domains. These domains are also known as PODs (PML Oncogenic Domain/Promyelocytic Oncogenic Domains). PML levels increase during both Rasinduced Senescence, leading to a dramatic increase in the size and number of PODs. Survival factors/Ras signaling induce Cellular Senescence by up-regulating PML gene expression though MAPK (Mitogen-Activated Protein Kinase) activation. PML is covalently modified and conjugated to SUMO1 (Small Ubiquitin Related Modifier-1). This enables PML to form NBs and enhances their interaction with other proteins. A specific dephosphorylation event triggered by As2O3 (Arsenic Trioxide) targets PML to the nuclear matrix to form Primary PML bodies. Sumolation then induces the maturation to Secondary PML bodies. In mature PML-NBs (or Secondary PML bodies), PML forms the outer shell and many proteins (Sp100, Rb, p53, Daxx, etc) are found within its electron clear core. DNA damage induced activation of p53-dependent Apoptosis requires PML. PML acts as a coactivator for p53 and increases acetylation of p53 by the transcriptional coactivator CBP (CREB-Binding Protein). This acetylation of p53 is reversed by Sirt (Sirtuin (Silent Mating Type Information Regulation-2 Homolog)) releasing p53 into p53 Pathway. PML associates with Daxx-mediated Apoptosis induced by Fas/FasL (Fas Ligand) and TNF (Tumor Necrosis Factor)/TNFR (Tumor Necrosis Factor Receptor) and regulates the transcriptional repressor activity of Daxx. PML acts with $\mathrm{Rb}$ and $\mathrm{p} 53$ to promote Ras-induced Senescence. PML-Sp100 NBs act against viral invasions. Mature PML-NBs are finally degraded by the 11S Proteasome Complex. 


\section{- Immunohistochemistry Stains}

Immunohistochemistry is not considered a molecular technique but it is based on the antigen-antibody affinity, it has emerged as a powerful investigative tool that can provide supplemental information to the routine morphological assessment of tissues. The use of immunohistochemistry to study cellular markers that define specific phenotypes has provided important diagnostic, prognostic, and predictive information relative to disease status and biology. The application of antibodies to the molecular study of tissue pathology (Fig.24) has required adaptation and refinement of immunohistochemical techniques, particularly for use in fixed tissues. In contrast to solution-based immunoassays that detect relatively abundant native proteins, in fixed tissues the preservation of antigen is variable and unpredictable. Thus, the history of immunohistochemistry has been a constant effort to improve sensitivity for detection of rare surviving antigenic targets with the ultimate goal of integrating tissue-based analysis with proteomic information.
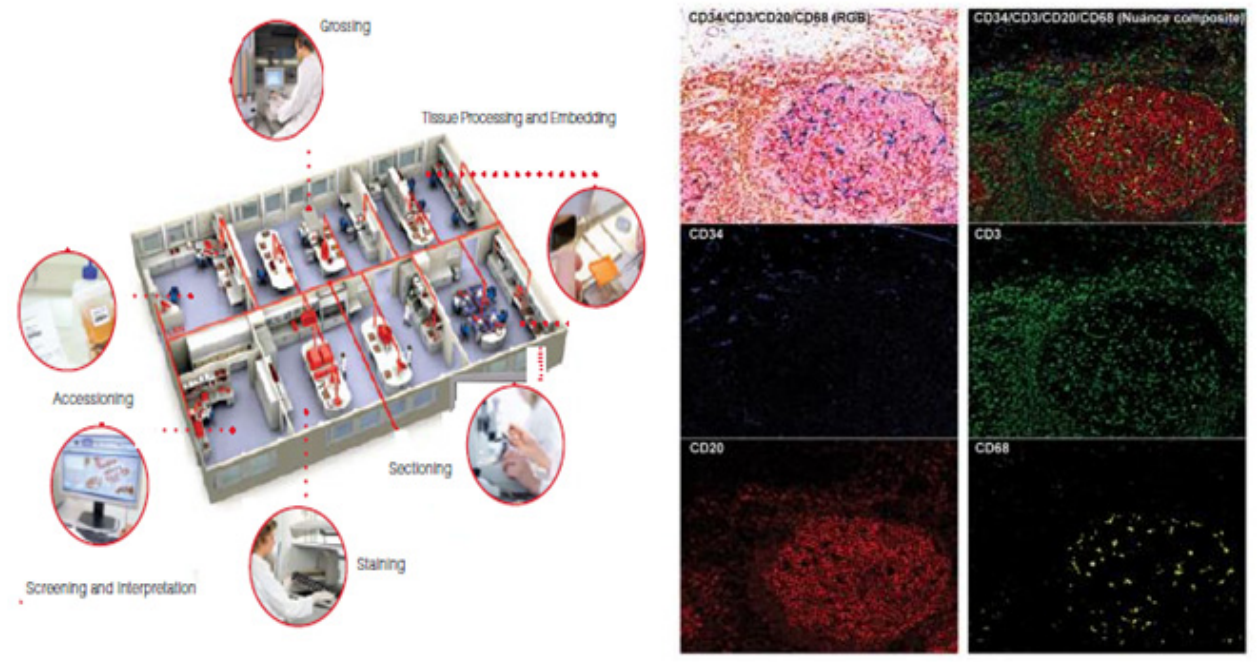

Figure 23. Immunohistochemistry Staining Stages.

- Nanotechnology in clinical laboratory diagnostics

Nanotechnologies enable diagnosis at the single-cell and molecule levels, and some can be incorporated in current molecular diagnostic methods, such as biochips. Nanoparticles, such as gold nanoparticles and quantum dots, are the most widely used, but various other nanotechnological devices for manipulation at the nanoscale as well as nanobiosensors are also promising for potential clinical applications, main applications of nanotechnology [43] .

Nanotechnologies will extend the limits of current molecular diagnostics and enable point-ofcare diagnostics, integration of diagnostics with therapeutics, and development of personalized medicine. Although the potential diagnostic applications are unlimited, the most important current applications are foreseen in the areas of biomarker discovery, cancer diagnosis, and 
detection of infectious microorganisms. Safety studies are needed for in vivo use. Because of its close interrelationships with other technologies, nanobiotechnology in clinical diagnosis will play an important role in the development of nanomedicine in the future.

\section{Author details}

Hussein A. Kaoud

Department of Hygiene and Environmental Pollution, Faculty of Veterinary Medicine, Cairo University, Giza, Egypt.

\section{References}

[1] Andersen MH, Schrama D, Thor Straten P, Becker JC. "Cytotoxic T cells". The Journal of Investigative Dermatology 2006; 126 (1): 32-41.

[2] Boon T, van der Bruggen P. "Human tumor antigens recognized by T lymphocytes". The Journal of Experimental Medicine 1996;183 (3): 725-9.

[3] Castelli C, Rivoltini L, Andreola G, Carrabba M, Renkvist N, Parmiani G . "T-cell recognition of melanoma-associated antigens". Journal of Cellular Physiology 2000;182 (3): 323-31.

[4] Romero P, Cerottini JC, Speiser DE. "The human T cell response to melanoma antigens". Advances in Immunology. Advances in Immunology 2006;92: 187-224

[5] Guevara-Patiño JA, Turk MJ, Wolchok JD, Houghton AN (2003). "Immunity to cancer through immune recognition of altered self: studies with melanoma". Advances in Cancer Research. Advances in Cancer Research 90: 157-77.

[6] Renkvist N, Castelli C, Robbins PF, Parmiani G. "A listing of human tumor antigens recognized by T cells". Cancer Immunology, Immunotherapy 2001;50 (1): 3-15.

[7] Gerloni M, Zanetti M. "CD4 T cells in tumor immunity". Springer Seminars in Immunopathology 21005; 27 (1): 37-48.

[8] Seliger B, Ritz U, Ferrone S. "Molecular mechanisms of HLA class I antigen abnormalities following viral infection and transformation". International Journal of Cancer 2006;118 (1): 129-38.

[9] Hayakawa Y, Smyth MJ. "Innate immune recognition and suppression of tumors". Advances in Cancer Research 2006;95: 293-322.

[10] Seliger B . "Strategies of tumor immune evasion". BioDrugs 2005; 19 (6): 347-54.

[11] Frumento G, Piazza T, Di Carlo E, Ferrini S. "Targeting tumor-related immunosuppression for cancer immunotherapy". Endocrine, Metabolic \& Immune Disorders Drug Targets 2006; 6 (3): 233-7.

[12] Stix, Gary. "A Malignant Flame" (PDF). Scientific American 2007;297 (1): 60-67.

[13] Erickson HS, Gillespie JW, Emmert-Buck MR. Tissue microdissection. Methods Mol Biol. 2008;424:433-48.

[14] Pagedar NA, Wang W, Chen DH, Davis RR, Lopez I, Wright CG, Alagramam KN. Gene expression analysis of distinct populations of cells isolated from mouse and human 
inner ear FFPE tissue using laser capture microdissection--a technical report based on preliminary findings. Brain Res.2006;1091(1):289-99.

[15] Huang Q, Sacks PG, Mo J, McCormick SA, Iacob CE, Guo L, Schaefer S, Schantz SP. A simple method for fixation and microdissection of frozen fresh tissue sections for molecular cytogenetic analysis of cancers. Biotech Histochem 2005; 80(3-4):147-56.

[16] Shibutani M, Uneyama C. Methacarn fixation for genomic DNA analysis in microdissected cells. Methods Mol Biol 2005; 293:11-25.

[17] Erickson HS, Gillespie JW, Emmert-Buck MR. Tissue microdissection. Methods Mol Biol 2008;424:433-48.

[18] Kryndushkin DS, Alexandrov IM, Ter-Avanesyan MD, Kushnirov VV. "Yeast [PSI+] prion aggregates are formed by small Sup35 polymers fragmented by Hsp104". Journal of Biological Chemistry 2003;278 (49): 49636-43.

[19] Sambrook J, Russel DW. Molecular Cloning: A Laboratory Manual 3rd Ed. Cold Spring Harbor Laboratory Press 2001. Cold Spring Harbor, NY.

[20] Berg JM, Tymoczko JL Stryer L. Biochemistry (5th ed.) 2002. WH Freeman.

[21] http://www.answers.com/topic/gel-electrophoresis\#ixzz1viquzJx9

[22] Liotta L, Kohn EC, Petricoin EF. Clinical proteomics: personalized molecular medicine. JAMA.2001;286:2211-2214.

[23] Lau AT, He QY, Chiu JF. Proteomic technology and its biomedical applications. (Shanghai) 2003;35: 965-975.

[24] Paweletz CP, Trock B, Pennanen M, et al. Proteomic patterns of nipple aspirate fluids obtained by SELDI-TOF: potential for new biomarkers to aid in the diagnosis of breast cancer. Dis Markers 2001;17:301-307.

[25] Issaq HJ, Veenstra TD, Conrads TP, Felschow D. The SELDI-TOF MS approach to proteomics: protein profiling and biomarker identification. Biochem Biophys Res Commun 2002;292:587-592.

[26] Copyright 2002 American Cancer Society. Reprinted with permission of Wiley-Liss, Inc, a subsidiary of John Wiley \& Sons, Inc.

[27] http://www.wisegeek.com/what-is-oncology.htm

[28] UCLA diagnostic molecular pathology laboratory, Department of Pathology and Laboratory Medicine, David Geffen School of Medicine.

[29] J Neurol Neurosurg Psychiatry (2000);68:521-525 doi:10.1136/jnnp.68.4.521

[30] BioTechniques, Vol. 50, No. 3, March 2011, pp. 182-186

[31] Myers RH. "Huntington's Disease Genetics". NeuroRx 2004;1 (2): 255-62.

[32] Walker FO. "Huntington's disease". Lancet 2007;369 (9557): 218-28.

[33] Hayden MR. "Predictive testing for Huntington's disease: a universal model?". Lancet Neurol 2003; 2 (3): 141-2.

[34] Fatima Smagulova, Ph.D., USU, and Kevin Brick, Ph.D., NIDDK, NIH.

[35] Leukemia 26, 708-715 (April 2012) | doi:10.1038/leu.2011.255

[36] haematol January 1, 2008 vol. 93 no. 134-40

[37] Scott LM, Tong W, Levine RL, Scott MA, Beer PA, Stratton MR, Futreal PA, Erber WN, McMullin MF, Harrison CN, Warren AJ, Gilliland DG, Lodish HF, Green ARJAK2 exon 
12 mutations in polycythemia vera and idiopathic erythrocytosis. $\mathrm{N}$ Engl J Med. 2007;356(5):459-68.

[38] Oki, E.; Oda, S.; Maehara, Y.; Sugimachi, K. "Mutated gene-specific phenotypes of dinucleotide repeat instability in human colorectal carcinoma cell lines deficient in DNA mismatch repair". Oncogene 1999; 18 (12): 2143-2147.

[39] https://www.pathnet.medsch.ucla.eud/referral/ODTCenter

[40] Torre D. Multiple sebaceous tumors. Arch Dermatol. 1968;98 (5):549-51.

[41] Heavey PM, McKenna D, Rowland IR. Colorectal Cancer and the Relationship Between Genes and the Environment. Nutr Cancer. 2004;48(2):124-141.

[42] https://www.qiagen.com/geneglobe/pathwayview.aspx?pathwayID=381\&ID=NM_0009 64,NM_016152,NM_000966

[43] Kewal K. Jain. Nanotechnology in clinicallaboratorydiagnostics. Clinica Chimica Acta 2005; Volume 358, Issues 1-2, Pages 37-54 